\title{
Chondrodysplasia Punctata
}

\author{
Narang $\mathrm{G}^{1}$, Arora $\mathrm{S}^{2}$, Khurana $\mathrm{MS}^{3}$, Singh $\mathrm{T}^{4}$, Shifali ${ }^{5}$
}

\section{Abstract}

We report a case of two and a half months old male child affected by chondrodysplasia punctata, a rare condition characterized by depressed nasal bridge, hypoplastic distal phalanges and epiphyseal stippling of the bones.

\section{Introduction}

C hondrodysplasia punctata is a group of dysplasias characterized by stippled calcifications within the epiphyses in infancy and associated with short stature, dry and scaly skin, occasional heart defects, and cataracts.

The most common form of chondrodysplasia punctata is Conradi-Hünermann syndrome, which is inherited as an X-linked dominant trait (CDPX2), also known as Conradi-HünermannHapple syndrome, is seen in girls and is a result of a mutation in the emopamil-binding protein $(E B P)$ gene. It is characterised by accumulation of calcium salts in long ends of bones, limb and joint shortening, flat face, thick scales, flaky skin, sparse coarse hair, alopecia, kyphosis and scoliosis.

A rarer autosomal recessive form, characterized by a rhizomelic pattern of dwarfism, is often fatal within the first year of life. It results from a variety of mutations in the PEX7 gene, which encodes the protein peroxin 7. This type of chondrodysplasia is genetically a member of the family of peroxisomal disorders, which include Zellweger syndrome and infantile Refsum disease. This form of disease is characterised by systemic shortening of limbs(rhizomelia), recurrent respiratory tract infections, seizures and congenital cataracts.

The X-linked recessive form of chondrodysplasia (CDPX1) has been localized to the arylsulfatase $\mathrm{E}$ gene (ARSE gene lies in short arm of $\mathrm{x}$ chromosome).Abnormalities in the ARSE gene lead to deficiency of a sulfatase enzyme that results in a high degree of sulfation in the cartilage matrix. This form occours exclusively in males and is characterized by deafness, skin and hair involvement, maxillary hypoplasia (flat nose), short columella, short ends of finger tips and toes.
'Dr. Gursharan Singh Narang, MBBS, MD, Professor, ${ }^{2}$ Dr. Sunita Arora, MBBS, MD, Associate Professor, ${ }^{3}$ Dr. Mandeep Singh Khurana, MBBS, MD, Associate Professor, ${ }^{4}$ Dr. Tarsem Singh, MBBS, MD, Assistant Professor, ${ }^{5} \mathrm{Dr}$. Shifali, MBBS, MD Resident. All from Sri Guru Ram Das Institute of Medical Science and Research, Vallah, Amritsar, India.

\section{Address for correspondence \\ Dr. Gursharan Singh Narang \\ E-mail: gsnarang321@gmail.com}

\section{How to cite}

Narang G, Arora S, Khurana MS, Singh T, Shifali5. Chondrodysplasia Punctata. J Nepal Paediatr Soc 2014;34(1):57-61.

doi: http://dx.doi.org/10.3126/jnps.v34i1.9110

This work is licensed under a Creative Commons Attribution 3.0 License.

(c) (i)

\section{The Case}

A two and a half months old male child presented to us with respiratory distress and oxygen dependency since soon after birth. The baby was born preterm by normal vaginal delivery. He was third in birth order, with two elder sisters who were normal. There was no history of still births or abortions. There was history of stridor along with one episode of cyanosis with respiratory distress soon after birth. On examination, the weight was appropriate for age. Bilateral air entry was equal and normal, subcostal recessions were present even on oxygen by nasal prongs. 
On examination baby had depressed nasal bridge due to hypoplasia of nasal bone and hypoplasia of distal phalanges of the hands, ichthyosis, irregular foci of alopecia, shortened neck, flattened facies with saddle nose, permanently flexed knee and elbow joints, limbs shortening, microcephaly and micrognathia (Fig 1 and 2). No other features of chondrodysplasia punctata like congenital dislocation of hip, undue rigidity of the larynx or trachea were noted. Chest skiagram showed calcification of trachea and bronchi (Fig 3). Infantogram showed stippled calcification of epiphyses of knee, hip, shoulder, wrist and vertebral bodies (Fig 4). There were no calcium deposits in the kidneys or the cartilages of the ears and nose. CT scan chest also revealed calcification of trachea (Fig 5)

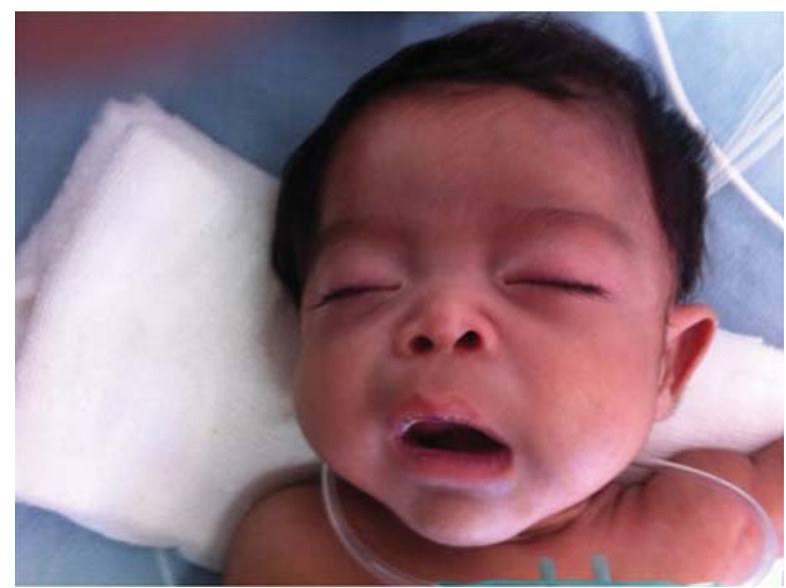

Fig1: Showing depressed nasal bridge

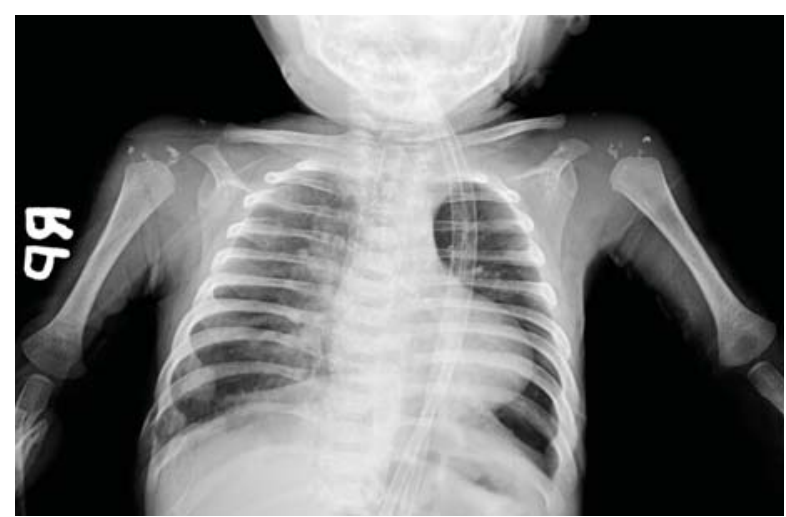

Fig 3: Stippled calcification of epiphysis of shoulder joint, trachea and bronchi.
Biochemical investigations like total and ionised serum calcium, phosphorus and alkaline phosphatase were within normal limits. Vitamin D levels were however below the normal levels.

It was possible, therefore, to exclude hypervitaminosis D or a general metabolic disorder, as a cause of ectopic calcification. Based on epiphyseal stippling, distal phalangeal hypoplasia in hands, mid facial hypoplasia, a presumptive diagnosis of chondrodysplasia punctata was thought of and venous blood sample of baby was sent for karyotyping which was reported as normal.

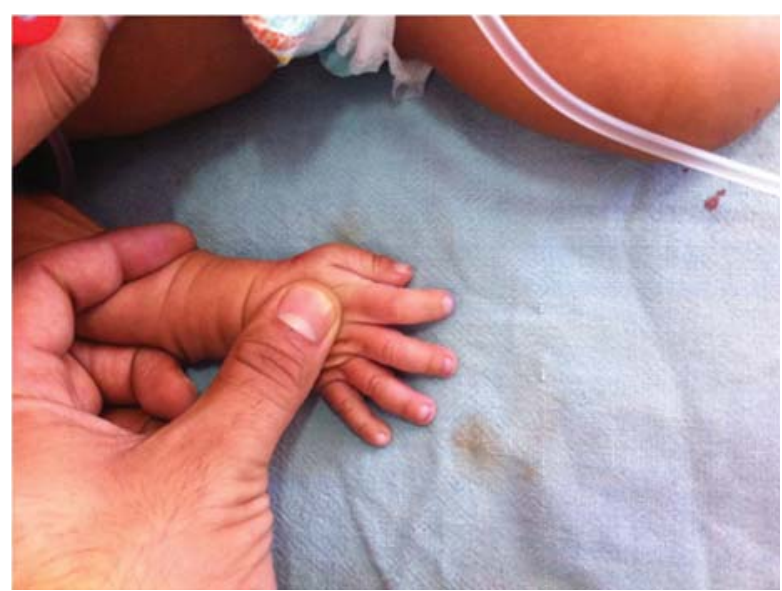

Fig 2: Showing hypoplasia of distal phalanges

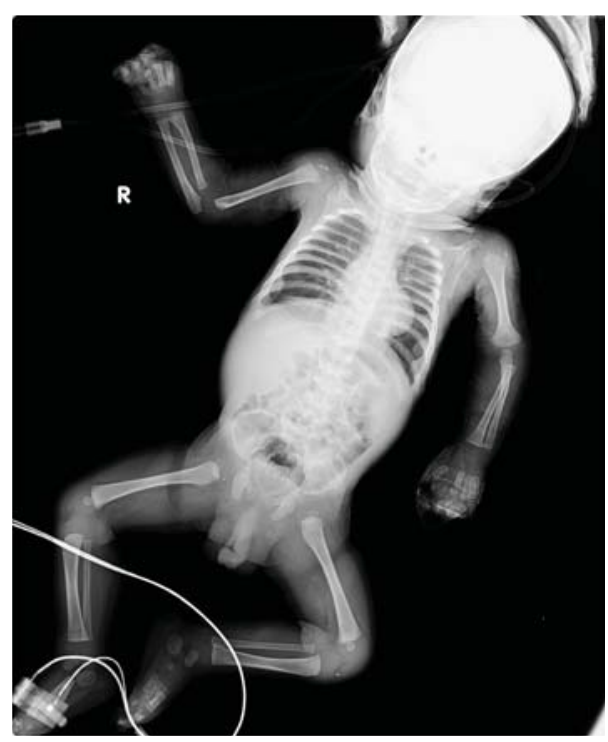

Fig 4: Infantogram showing stippled calcification of epiphyses of knee, hip, shoulder, wrist and vertebral bodies. 


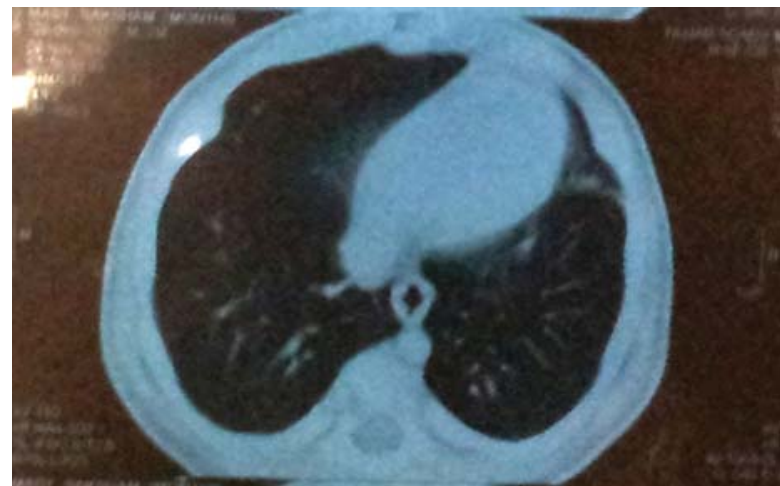

Fig 5: CT scan of upper chest showing tracheal calcification

\section{Discussion}

Bone dysplasias are characterized by changes in growth, cartilaginous and bone development, as well as in bone remodeling ${ }^{1}$, mainly the $\mathrm{X}$-linked dominant (Conradi-Hünnermann syndrome) and autosomal recessive rhizomelic forms of the disease have been described.

The chondordysplasia punctata (CDP) dominant form is most frequent and is related to a defect in peroxisomal enzyme biosynthesis. The clinical picture may range from mild disease to an extremely severe condition, with cataracts, asymmetrical shortening of long bones, scoliosis, ichthyotic-type skin lesions, and flattened facies with broad nasal bridge ${ }^{2}$. There is some life expectancy, although early fetal death can occur. Infants who survive beyond the first year usually present normal life expectancy and mental development ${ }^{3}$.

The rhizomelic CDP is of autosomal recessive origin, characterized by a peroxisomal functional defect resulting in an enzymatic deficiency where there is a decrease in the plasmalogen synthesis, decrease in phytanic acid oxidation and presence of a unprocessed (inactive), the 3-oxacyl-CoA-thiolase ${ }^{4}$.

Currently this form of CDP is diagnosed through clinical features compatible with the syndrome associated with biochemical findings including serum phytanic acid levels, screening of plasmalogen synthesis on cultured fibroblasts ${ }^{4}$, as well as in erythrocytes, and plasmatic level of fatty acids with long carbon chain ${ }^{4,5}$. The plasma level of phytanic acid is high, and the plasmalogen synthesis in fibroblast and erythrocytes is reduced usually, the serum level of fatty acids with long carbon chain is normal $5,6,7,8$. The chromosomal study demonstrates PEX7 mutation, $50 \%$ of them in the L292ter allele 4 . None of these biochemical or genetic tests has been performed in the present case.
This CDP variant is rare, with an estimated incidence of 1:100,000 and with only 72 cases reported in the literature up to $1995^{2,9}$.

The main characteristics described in the literature are symmetric and severe rhizomelicmicromelia (proximal shortening of limbs), punctate calcifications and long bones metaphyseal and epiphyseal ossification changes, punctate calcifications and coronal clefts in vertebral bodies of the thoracic and lumbar spine, microcephaly and growth retardation, psychomotor delay, spasticity and precocious death ${ }^{2,4,10}$. The presence of vertebral clefts, radiotransparent longitudinal zone observed in the lateral view, previously described as invariable in cases of rhizomelic CDP has not been present in three of five cases analyzed by Wardinsky et al and in other cases reported in the literature, and is not obligatorily necessary for the diagnosis ${ }^{5}$.

Other characteristics have been described with a variable frequency like ichthyosis, cataracts, restricted joint mobility, sucking and deglutition difficulty, alopecia, auditive and visual dificiencies, seizures, optic nerves hypoplasia, kyphoscoliosis and cleft spine $e^{3,4,11,12}$. Patients usually present with flattened facies with micrognathia, malar hypoplasia, flattened nasal bridge and bulbous nose. In contrast to the other forms of CDP, the rhizomelic CDP presents a poor prognosis, with repetitive infections and death in the first two years of life l, $^{2,5,6}$.

The differential diagnosis includes other causes for CDP, Keutel syndrome (cartilage calcification in ears, nose larynx, ribs, pulmonary artery stenosis, brachytelephalangism), Zellweger syndrome (hypotonia, poor feeding, liver cysts with hepatic dysfunction), Smith-Lemli-Opitz syndrome (dysmorphic facial features, microcephaly, second and third toe syndactyly, MR), classical and neonatal Refsum disease(neurologic dysfunction, cerebellar degeneration, peripheral neuropathy and onset in childhood), neonatal adrenoleucodystrophy (severe hypotonia, hepatomegaly, retinitis pigmentosa), congenital infections, and maternal use of coumarinlike compounds or phenytoin during gestation (fetal hydantoin syndrome-facial features, growth developmental delay, underdeveloped nails) ${ }^{2,4,6,9}$.

Our patient presented ichthyosis, irregular foci of alopecia, shortened neck, flattened facies with saddle nose, permanently flexed knee and elbow joints, microcephaly and micrognathia and history of deglutition difficulty, clinical characteristics corroborating the diagnosis of rhizomelic CDP. An usual characteristic of the rhizomelic CDP is the presence 
of coronal clefts in vertebral bodies. In the present case, coronal clefts in L2 and L5 vertebral bodies were identified, besides proximal, symmetrical bilateral shortening of upper and lower limbs bones with punctate calcifications on the long bones epiphysis. These clefts are a result of poor fusion of anterior and posterior halves of vertebral bodies, occurring around the fourth month of gestation $n^{4,5,13}$. Punctate calcifications are caused by a progressive cartilage degeneration represented by chondrocytes with a pycnotic nucleus and eosinophil cytoplasm followed by ossification ${ }^{13}$.

Auditory and/or visual dificiencies have been observed, although being found with variable frequency in the referred literature. However, another typical finding in this type of chondrodysplasia is microcephaly, which has shown to be quite accentuated, considering the values very below than normal. Considering the exuberance of the clinical-radiological picture in the index case, a diagnosis of recessive CDP was made.

It is important to note that patients with diagnosis of rhizomelic CDP should undergo ambulatorial followup, as, in spite of the current inexistence of specific treatment, many clinical manifestations, like alopecia, ichthyosis and cataracts, might not be present at the moment of the diagnosis, showing up with the progress of the disease. Other manifestations, like punctate calcifications, tend to disappear with aging, without resulting in bone deformities. Presently, the patient is being kept in ambulatory follow-up in our institution.

CDP is a very rare disorder with characteristic features. Some of the manifestations present later with progress of disease others like punctuate calcification tend to disappear with aging. Since no specific treatment is available these should be kept on regular follow up. Previously, antenatal diagnosis of this condition was made by radiography. The prenatal diagnosis of chondrodysplasia punctata has been made with ultrasonography. The punctate calcifications can be seen in late pregnancy in the rhizomelic form, whereas limb shortening is apparent earlier, specifically stippling of the proximal humerus, has permitted us to identify the condition in a fetus at 28 weeks' gestation ${ }^{14}$. Amniocentesis has also been useful in diagnosing the rhizomelic form.

\section{Conclusion}

Chondrodysplasia Punctata is a rare disorder which could be missed in routine examination of children. Since there is no treatment these children should be kept on a regular follow up because some of the manifestations could disappear with time. Definitive diagnosis helps in planning future pregnancies from the point of view of antenatal diagnosis and genetic counseling.

\section{References}

1. Fairbank HAT: Dysplasia epiphysialis punctata; synonyms: stippled epiphyses, chondrodystrophia calcificans congenita (Hunermann). J Bone Joint Surg Br 1949;31B(1):114-22.

2. OmobonoE, Goetsch W. Chondrodysplasiapunctata (the Conradi-Hünnermann syndrome). A clinical case report and review of the literature. Minerva Pediatr 1993;45:117-121.

3. Labrunie E, Pereira LF, Guimarães RR. Displasiaepifisáriapunctata - relato de caso. Radiol Bras 1999;23:161-164.

4. Pascolat G, Zindeluk JL, Abraão KC, Rodrigues FM, Guedes CIM. Condrodisplasiapuntiforme forma rizomélica - relato de caso. J Pediatr 2003; 79:189-192.

5. Wardinsky TD, Pagon RA, Powell BR, et al. Rhizomelicchondrodysplasiapunctata and survival beyond one year: a review of the literature and five case reports. Clin Genet 1990;38:84-93.

6. Kumada S, Hayashi M, Kenmochi J, et al. Lethal form of chondrodysplasiapunctata with normal plasmalogen and cholesterol biosynthesis. Am J Med Genet 2001;98:250-255.

7. Ikegawa S, Ohashi H, Ogata T, et al. Novel and recurrent EBP mutations in X-linked dominant chondrodysplasiapunctata. Am J Med Genet 2001;94:300-305.

8. Heymans HS, Oorthuys JW, Nelck G, Wanders RJ, Schutgens RB. Rhizomelicchondrodysplasiapunctata: another peroxisomal disorder [letter]. $N$ Engl J Med 1985;313:187-188.

9. Fourie DT. Chondrodysplasiapunctata: case report and literature review of patients with heart lesions. Pediatr Cardiol 1995;16:247-250.

10. Spranger JW, Opitz JM, Bidder U. Heterogeneity of chondrodysplasiapunctata[abstract]. Humangenetik 1971;11:190-212.

11. Agamanolis DP, Novak RW. Rhizomelicchondrodysplasiapunctata: report of a case with review of the literature and correlation with other peroxisomal disorders. Pediatr Pathol Lab Med 1995; 15:503-513. 
12. JansenV,SarafoglouK, RebarberA, GrecoA, Genieser NB, Wallerstein R. Chondrodysplasiapunctata, tibial-metacarpal type in a 16 week fetus. J Ultrasound Med 2000;19:719-722.

13. Poulos A, Sheffield L, Sharp $P$, et al. Rhizomelicchondrodysplasiapunctata: clinical, pathologic, and biochemical findings in two patients. J Pediatr 1988;113:685-690.

14. Argo KM, Toriello HV, Jelsema RD, et al.: Prenatal findings in chondrodysplasia punctata, tibiametacarpal type. Ultrasound Obstet Gynecol 1996;8(5):350-4. 